\title{
El cálculo diferencial e integral en una variable en la formación inicial de docentes de matemática en Costa Rica
}

Fonseca Castro, Jennifer Liseth1; Alfaro Carvajal 2, Cristian Roberto

El cálculo diferencial e integral en una variable en la formación inicial de docentes de matemática en Costa Rica

Revista Educación, vol. 42, núm. 2, 2018

Universidad de Costa Rica, Costa Rica

Disponible en: http://www.redalyc.org/articulo.oa?id=44055139017

DOI: https://doi.org/10.15517/revedu.v42i2.25844

Esta obra está bajo una Licencia Creative Commons Atribución-NoComercial-SinDerivar 3.0 Internacional. 


\section{El cálculo diferencial e integral en una variable en la formación inicial de docentes de matemática en Costa Rica}

\section{Differential and Integral Calculus in a Variable in the Training of Teachers of Mathematics in Costa Rica}

Jennifer Liseth1 Fonseca Castro [1]

Universidad Nacional, Costa Rica

jennifer.fonseca.castro@una.cr

Cristian Roberto Alfaro Carvajal 2 [2]

Universidad Nacional, Costa Rica

cristian.alfaro.carvajal@una.cr
DOI: https://doi.org/10.15517/revedu.v42i2.25844

Redalyc: http://www.redalyc.org/articulo.oa?id=44055139017

\section{ReSUMEN:}

El cálculo diferencial e integral está presente en la mayoría de los planes de estudios de distintas carreras universitarias vinculadas a las ciencias exactas y naturales en Costa Rica. Las reflexiones sobre el qué, cómo y para qué enseñar cálculo han estimulado el estudio de esta área desde distintas aristas. En Costa Rica, personal académico e investigador de las universidades estatales encargadas de la formación de docentes de matemática para el futuro, se han dado a la tarea de revisar y plantear modificaciones a los cursos de matemática, en particular los de cálculo diferencial e integral en una variable. En este sentido, este trabajo resume las perspectivas de autoridades administrativas de las escuelas de matemática de las universidades antes mencionadas, del personal docente de matemática de dichas instituciones con experiencia en la enseñanza del cálculo diferencial e integral y de estudiantado avanzado de la carrera de Enseñanza de la Matemática de la Universidad Nacional (UNA) de Costa Rica sobre los propósitos, el enfoque, la metodología y los temas de mayor dificultad en el proceso de enseñanza-aprendizaje del cálculo diferencial e integral en una variable en la preparación de docentes de matemática en formación inicial. Los datos se obtuvieron mediante entrevistas, cuestionarios y talleres con las autoridades administrativas de las escuelas de matemática de las universidades estatales, así como con el personal docente de matemática de dichas instituciones y estudiantado avanzado de la carrera de Enseñanza de la Matemática de la UNA. En cuanto a los principales resultados, se detectaron como temas con mayor dificultad en la enseñanza-aprendizaje del cálculo diferencial e integral aquellos en los existían mayores requerimientos conceptuales y de razonamiento matemático, por ejemplo, los problemas de optimización y de razones de cambio relacionadas; así como la demostración de teoremas. En relación con el uso de resolución de problemas, el personal docente lo considera importante en la construcción de conceptos matemáticos; no obstante, lo enfoca en la práctica de forma rutinaria, al finalizar un tema. Se espera que los insumos de esta investigación sirvan de fundamento en las reformas curriculares en la formación de las futuras generaciones de docentes de matemática.

Palabras clave: cálculo diferencial, cálculo integral en una variable, formación de generaciones futuras de docentes de matemática, resolución de problemas.

\section{Abstract:}

The differential and integral calculus is present in most of the curricula of various university careers of the exact and natural sciences in Costa Rica. Reflections and discussions about the what, how, and what to teach of calculus have stimulated the study of it from different perspectives. In Costa Rica, academics and researchers from the state universities responsible of the formation of future teachers have been given the task to review and revise their mathematics courses, particularly those related to differential and

\section{NoTAS DE AUTOR}

[1] Maestría en Enseñanza de la Matemática de la Universidad de Purdue, Indiana, USA, excoordinadora de la Carrera de Bachillerato y Licenciatura en la Enseñanza de la Matemática de la Escuela de Matemática de la Universidad Nacional, actualmente académica de la Escuela de Matemática de la Universidad Nacional, cuenta con publicaciones en el área de la matemática educativa en revistas tales como UNICIENCIA, Cuadernos de Investigación y Formación en Educación Matemática, Tercer Informe del Estado de la Educación, Revista Unión en España, North American Chapter International Group for the Psychology of Mathematics Education.

[2] Maestría en Matemática con Énfasis en Matemática Educativa de la Universidad de Costa Rica, exsubdirector de la Escuela de Matemática de la Universidad Nacional, actualmente académico de la Escuela de Matemática de la Universidad Nacional, cuenta con publicaciones en el área de la matemática educativa en revistas tales como UNICIENCIA, Cuadernos de Investigación y Formación en Educación Matemática. 
integral calculus in one variable. In this sense, this article summarizes the perspectives of administrative authorities of the School of Mathematics at the aforementioned universities, mathematics professors from such institutions with experience in teaching calculus in one variable and advanced students of the Mathematics Teaching major at Universidad Nacional de Costa Rica (UNA) about the purposes, the approach, the methodology, and the most difficult issues in the process of teaching and learning differential and integral calculus in one variable in the training of mathematics teachers. Data was obtained from interviews and questionnaires applied as well as workshops for administrative authorities and mathematics professors from the School of Mathematics at public universities and advanced students pursuing the Mathematics Teaching major at UNA. Regarding the main results, the subjects with greater difficulty in the teaching-learning of differential and integral calculus were detected. Those in which there were greater conceptual requirements and mathematical reasoning; for example, optimization problems and related reasons for change as well as the proof of theorems. In regards to the use of problem solving, teachers consider it important in the construction of mathematical concepts; however, they focus on practicing it regularly at the end of a topic. It is expected that this research will provide a foundation for the curricular reforms in the formation of future mathematics teachers.

KEYWORDS: differential and integral calculus in one variable, mathematics, problem solving, teacher training.

\section{INTRODUCCIÓN}

La matemática ocupa un sitio preponderante en el desarrollo de las sociedades actuales. El rápido crecimiento de las ciencias y las tecnologías exige la formación de profesionales con competencias matemáticas y científicas para afrontar los nuevos retos que impone un mundo más globalizado y competitivo. Desde esta perspectiva, la educación superior juega un rol primordial en el desarrollo social, cultural y económico del país, por tanto, requiere de personal docente de matemática altamente calificado, con sólidos conocimientos matemáticos y con herramientas pedagógicas y tecnológicas adecuadas, que permitan el desarrollo integral en sus estudiantes.

En la educación pública costarricense, las Escuelas de Matemática[3] de la Universidad Nacional (UNA), la Universidad de Costa Rica (UCR), el Instituto Tecnológico de Costa Rica (ITCR) y la Universidad Estatal a Distancia (UNED) son las instituciones encargadas de la formación de docentes de matemática del país. En este sentido, estas asumen retos que coadyuvan en la formación de profesionales en la enseñanza de la matemática en concordancia con las nuevas perspectivas de desarrollo; asimismo, potencian esfuerzos para la generación de ofertas académicas pertinentes. Los distintos procesos de autoevaluación de las carreras en enseñanza de la matemática de dichas instituciones han permitido la reflexión y discusión de aspectos a lo que debe enseñarse y la manera en la que debe hacerse en cada una de las áreas disciplinarias de sus carreras. Un área que ha sido de interés en dichas reflexiones, dada la complejidad de sus conceptos, es la enseñanza y aprendizaje del cálculo diferencial e integral.

Desde la inclusión del cálculo diferencial e integral en los currículos escolares, ha habido diferentes enfoques sobre la forma en que este se debe enseñar (Depool, 2005). Por ejemplo, hay quienes se inclinan por la enseñanza de procedimientos algorítmicos, los cuales permitan la solución de problemas rutinarios. Otros grupos enfatizan en las demostraciones rigurosas y el formalismo matemático (Tall, 1997). Pese a que los avances en la computación han estimulado el uso de software para la enseñanza-aprendizaje del cálculo diferencial e integral, algunas de las propuestas educativas quedan reducidas al desarrollo de procedimientos algebraicos, en lugar de la construcción de conocimientos (Depool, 2005).

Así, se hace necesario repensar la manera en que se enseña el cálculo diferencial e integral, desde los contenidos planteados, hasta la forma en que estos se estructuran y abordan (Salinas y Alanís, 2009). En este sentido, las discusiones deben girar en torno a cuál debe ser la preparación en esta área para docentes de matemática en preparación inicial. De esta manera, cobran relevancia las siguientes preguntas: ¿cuáles son los contenidos del cálculo diferencial e integral en una variable que se deben desarrollar en la preparación de docentes de matemática en formación inicial?, ¿con qué profundidad deben ser abordados estos contenidos matemáticos?, ¿cuáles son los enfoques metodológicos que favorecen la enseñanza de estos contenidos matemáticos?, ¿cuál es el propósito del cálculo diferencial e integral en su formación? 
En consecuencia, se hace fundamental conocer la perspectiva de los principales actores en la preparación de docentes de matemática, sobre los procesos de enseñanza-aprendizaje de la línea del cálculo diferencial e integral, para abordar con mayor fundamento las cuestiones del desarrollo profesional de estas generaciones docentes (Nava y Reyes, 2009).

$\mathrm{Al}$ respecto Ruthven (2002) afirma:

Extraer y codificar este conocimiento artesanal [el de los docentes] tiene el potencial de mejorar la efectividad con que se puede realizar la formación de profesores, proporcionando marcos más explícitos para analizar los procesos de enseñanza, para articular mecanismos y funciones, y para comprender su adaptación a condiciones diferentes. (p. 596)

El objetivo de este trabajo es determinar las perspectivas de autoridades de las escuelas de matemática de la UNA, UCR, ITCR y UNED, del personal docente de matemática de dichas escuelas con experiencia en la enseñanza del cálculo diferencial e integral y de estudiantado avanzado de la carrera de Bachillerato y Licenciatura en la Enseñanza de la Matemática de la UNA, sobre los propósitos, el enfoque, la metodología y los temas de mayor dificultad en el proceso de enseñanza-aprendizaje del cálculo diferencial e integral en una variable en la preparación de docentes de matemática en formación inicial. Para ello, se aplicó un cuestionario al profesorado de matemática, una entrevista a las autoridades y un taller con el estudiantado avanzado de dicha carrera.

\section{MARCo té́RICO}

La enseñanza del cálculo diferencial e integral en una variable en la formación de docentes de matemática

Ball, Thames y Phelps (2008) señalan que docentes de matemática deben tener el conocimiento matemático necesario para llevar a cabo su labor profesional. Esto requiere un docente o una docente capaz de llevar a la práctica las exigencias planteadas en el currículo. Particularmente, el Ministerio de Educación Pública (MEP) costarricense establece, en el Programa de Estudios de Matemáticas (MEP, 2012) el desarrollo de competencias en la resolución de problemas y en procesos de razonamiento y argumentación.

El área del cálculo diferencial e integral favorece el planteamiento de problemas que requieren la formulación de conjeturas, de procesos inductivos, de argumentación y validación del conocimiento matemático (Fischbein, 1994). En este sentido, el cálculo aporta herramientas de conocimientos y habilidades a docentes de matemática en formación inicial, esenciales para su desarrollo profesional.

Lo anterior está en concordancia con los objetivos generales de la enseñanza de la matemática, los cuales incluyen preparar a la persona para la ciudadanía, servir de base para una carrera de ciencia y tecnología, así como estimular la creatividad (D'Ambrósio, 2002).

Se requiere de estrategias metodológicas para la enseñanza del cálculo diferencial e integral que favorezcan los procesos de construcción de conceptos, generación de conjeturas, validación y refutación; en donde la introducción formal de los conceptos no sea el foco de la enseñanza.

Existen distintas posiciones sobre la forma de abordar el cálculo diferencial e integral. Al respecto, Artigue (1998) asegura que el desarrollo del cálculo depende de su evolución global y de las condiciones culturales y sociales, en las cuales se desarrolla el proceso de enseñanza-aprendizaje. En algunos casos, los conceptos son introducidos de manera intuitiva; en otros, por medio de la definición formal basada en las teorías de análisis matemático; existen también los que se quedan en una posición intermedia entre estos dos extremos. Resalta también la ampliación del significado de cálculo diferencial en integral desde las técnicas simbólicas tradicionales hasta una ciencia de cómo los objetos cambian (considerando su razón de cambio y el crecimiento acumulado) y la accesibilidad del cálculo al estudiantado (Artigue, 1998, citado por DosSantos, 2012).

El cálculo diferencial e integral presenta una serie de dificultades en la enseñanza-aprendizaje que deben ser consideradas para la formación de docentes de matemática. Dreyfus y Eisenberg (1990) afirman que el 
cálculo diferencial e integral es la rama de la matemática avanzada a la que se debe dedicar más tiempo en los estudios científico-técnicos, dado el número de problemas no triviales presentes en su proceso de aprendizaje. Existen "limitaciones tanto de las prácticas educativas tradicionales, como en las que favorecen los enfoques formales y teóricos" (Artigue, 1991, p.119). Los elevados niveles de fracaso escolar, de reprobación y deserción de estudiantes en cursos de cálculo revelan la complejidad existente en su estudio y la necesidad de desarrollar nuevas investigaciones en esta dirección (Artigue, 1991).

\section{Enfoques y metodologías para la enseñanza del cálculo diferencial e integral en una variable}

Las metodologías tradicionales en la enseñanza del cálculo diferencial e integral a nivel universitario se centran fundamentalmente en aspectos algebraicos y algorítmicos. Se abordan los procesos rigurosos de demostración matemática y la evaluación consiste en presentar al estudiantado ejercicios similares a los desarrollados en clase, de manera que tengan correspondencia con la estructura expuesta por el personal docente de matemática (Moreno, 2005).

Esta metodología presenta una serie de dificultades. En efecto, es posible que el conocimiento adquirido por el estudiantado le permita resolver problemas y ejercicios denominados rutinarios, es decir, situaciones que sean muy similares a las presentadas por su docente en la clase. No obstante, cuando el estudiantado afronta situaciones que requieren un mayor manejo conceptual, la mayoría comete errores (Selden, Mason y Selden, 1994).

Harel y Trgalová (1996) proponen tres enfoques (proyectos) en la enseñanza del cálculo diferencial e integral:

1. Proyecto de cálculo diferencial e integral en contexto: En este enfoque se plantea que el cálculo es un lenguaje, una red de conceptos y un conjunto de técnicas. La metodología consiste en el planteamiento de problemas y situaciones reales, el estudiantado trabaja en grupos pequeños. Los problemas se abordan inicialmente con métodos numéricos y con la ayuda de la computadora. Posteriormente, se plantean soluciones analíticas. De esta manera se construyen finalmente los conceptos del cálculo diferencial e integral.

2. Proyecto de debate científico: En este enfoque se introducen los conceptos del cálculo diferencial e integral mediante la presentación de problemas científicos, de este modo, el estudiantado trabaja como si fuese matemático, para ello, debe formular preguntas, hacer conjeturas y realizar discusiones con el resto de pares de la clase.

3. Ingeniería didáctica: Se propone una investigación que consta de tres etapas, a saber, análisis e interpretación de la enseñanza, análisis de las restricciones en la enseñanza; el diseño de una ingeniería didáctica.

Otro enfoque que ha surgido como una respuesta a las dificultades presentadas por parte de estudiantes en el proceso de enseñanza-aprendizaje es la resolución de problemas. Este enfoque es una línea de investigación en educación matemática, la cual ha sido relevante en los últimos tiempos. Su importancia ha sido creciente, debido a que muchos estudios consideran la resolución de problemas como la esencia de la matemática y el medio para lograr el aprendizaje en esta. Mediante su uso, el estudiantado debería potenciar ciertas habilidades tales como: razonamientos más adecuados, la persistencia, la curiosidad y el logro de mayor confianza para enfrentar situaciones no rutinarias, que trascienden incluso el ámbito escolar (Godino, 2010).

Este autor sostiene que la resolución de problemas es una pieza clave en cualquier proceso de aprendizaje matemático, por lo tanto, debe estar articulada en el proceso de estudio. Los problemas en este enfoque deben plantearse antes al estudiantado para favorecer la construcción de los conceptos matemáticos. Posteriormente, pueden usarse también para aplicarse en diferentes contextos. 


\section{Dificultades en la enseñanza y aprendizaje del cálculo diferencial e integral en una variable}

El cálculo diferencial e integral en una variable tiene como objeto de estudio las funciones, las cuales están presentes en todos los modelos matemáticos, lo cual hace indispensable su estudio en todas las ciencias básicas y sociales, así como en las ingenierías (Cuevas y Pluvinage, 2009).

Para quienes escriben este texto, una de las posibles causas del fracaso en el aprendizaje de esta disciplina es que su enseñanza está planteada en dos extremos. Por un lado, una enseñanza muy procedimental, la cual va en detrimento de los aspectos conceptuales. Por otro lado, una enseñanza basada en aspectos muy formales. En general, plantean que los cursos de cálculo se inician con enunciados, teoremas y problemas estándar, los cuales tienen como finalidad ilustrar los conceptos expuestos. El énfasis se da al manejo algebraico, más que a lo visual y lo geométrico.

En la mayoría de los programas de estudio curriculares de cálculo diferencial e integral se hace un estudio sobre los axiomas de campo y de orden del conjunto de los números reales. Seguidamente se hace el estudio de las funciones algebraicas, específicamente los límites, la continuidad, la derivación y las aplicaciones de la derivada. No obstante, históricamente el desarrollo de los conceptos fue muy diferente a la forma en la que se presentan en la actualidad tales contenidos. Por ejemplo, en los siglos XVIII y XIX el estudio completo de los números reales se relacionó con la resolución de problemas hallados al tratar funciones matemáticas. Al dejar de lado la historia del desarrollo del cálculo diferencial e integral, se pierde la gran oportunidad de explorar los problemas que le dieron origen (Cuevas y Pluvinage, 2009).

De acuerdo con estos autores, los cursos del cálculo diferencial e integral entre los siglos XVI y XIX se desarrollaban a partir de la siguiente secuencia:

Variaciones de polinomios y derivada de un polinomio.

1. Relación entre tangente a una curva y derivada (Barrow); derivada de las funciones algebraicas y nacimiento del cálculo (L'Hôpital).

2. Funciones trigonométricas y transcendentes (logaritmo y exponencial) y su derivada (Newton, Leibniz), desarrollos de Taylor y series.

3. Consideración general de funciones reales por Euler, sin poseer una definición formal del objeto función.

4. Estudio de problemas de convergencia de series y continuidad (Fourier).

5. Definición formal de los números reales y de la derivada en un punto como límite (Weierstrass).

Al comparar la secuencia anterior con el orden actual en el cual se desarrollan los cursos de cálculo diferencial e integral, existe una gran incoherencia, la cual podría ser una de las dificultades en el aprendizaje del cálculo diferencial e integral (Cuevas y Pluvinage, 2009). En este sentido, manifiestan que un primer curso de cálculo diferencial no debe basarse en la estructura formal de los números reales, ni del concepto de función. Tampoco es necesario construirlo sobre el concepto de límite, el cual debe surgir al final del curso. En este sentido, recomiendan usar ideas provenientes de la geometría o del estudio del movimiento.

Según Delgado (2009), existe un problema en el proceso de aprendizaje estudiantil en los cursos de cálculo o análisis denominado "Efecto frontera". Este consiste en la desaparición de los conceptos del cálculo, de tal manera que el aprendizaje se transforma en una reducción algebraica de los elementos básicos de esta disciplina. De este modo, se fomenta la creencia de que el objetivo fundamental del aprendizaje del cálculo diferencial e integral es la correcta manipulación simbólica.

Díaz (2009) afirma que en los cursos de cálculo diferencial e integral existe una tendencia a enfocarse en el desarrollo de habilidades en los aspectos mecánicos, así como en la memorización de algoritmos. En este sentido, manifiesta que lo anterior no contribuye a la comprensión de las ideas fundamentales del cálculo diferencial e integral, pues se relega el significado y la interpretación de los conceptos. Considera importante 
no trasladar la enseñanza del cálculo diferencial e integral al extremo teórico en donde el foco primordial sean los teoremas y sus demostraciones.

Otro aspecto fundamental en la enseñanza del cálculo diferencial e integral es el personal docente de matemática. El profesorado puede manejar las ideas del cálculo; sin embargo, entenderlas, manejarlas, interpretarlas y enseñarlas son procesos diferentes y complejos. Desde la premisa de que los conocimientos están ligados a las creencias, si al carácter verdadero de la creencia se le agregan justificaciones de esa veracidad, entonces, la creencia adquiere el estatus de conocimiento. A partir de lo anterior, solo existen conocimientos verdaderos, así, cuando se afirma conocer algo y ese algo es falso, entonces, ese algo no es un conocimiento; simplemente es una creencia falsa. De este modo, en el salón de clases no necesariamente se construye conocimiento, sino que en ocasiones es el lugar en donde se desarrollan las creencias del personal docente, y esto coadyuva a obstaculizar el aprendizaje del estudiantado (Díaz, 2009).

Para Ímaz y Moreno (2009), la enseñanza del cálculo diferencial e integral sufre una serie de problemáticas tales como:

- Un exceso de rigor al confundir al cálculo diferencial e integral con el análisis, en este sentido, mencionan a Klein, quien en el siglo XIX manifestó que el cálculo se podía entender mejor desde el análisis. Afirman que esta tesis es ampliamente compartida en los sectores académicos.

- La satanización de los infinitesimales, en este sentido, mencionan a Cantor, matemático alemán del siglo XIX, quien se refirió despectivamente a ellos.

- Una gran antipatía al estudio del cálculo diferencial e integral la cual es favorecida por la proliferación de los llamados "textos de cálculo", que consisten en grandes libros más preocupados por el negocio que por la academia.

Estos autores identifican tres tipos de dificultades:

a) Las que están asociadas a la complejidad matemática de los objetos básicos tales como los números reales, las funciones y las sucesiones.

b) Las que se asocian a la conceptualización del límite y a su manejo técnico.

c) Las asociadas a la ruptura con formas de pensamiento característico del funcionamiento algebraico.

Di Blasi Regner (2003, citado por Abrate, Pochulu y Vargas, 2006) hace la siguiente clasificación de las dificultades que pueden presentarse en el proceso de enseñanza-aprendizaje de la matemática en general:

1. Dificultades asociadas a la complejidad de los objetos matemáticos: la comunicación en matemática de forma escrita se lleva a cabo mediante el uso de los símbolos matemáticos. Al intentar dar una interpretación de los objetos matemáticos mediante el lenguaje natural se presentan problemas de precisión.

2. Dificultades asociadas a los procesos de pensamiento matemático: esta dificultad refiere a la incapacidad de seguir un argumento lógico.

3. Dificultades asociadas a los procesos de enseñanza: estas dificultades están relacionadas con tres elementos fundamentales, a saber, la institución en donde se lleva a cabo el proceso de enseñanzaaprendizaje, el currículo de matemática a desarrollar y los métodos de enseñanza empleados por el personal docente de matemática. La institución debe generar procesos de organización que tiendan a reducir las dificultades del aprendizaje de la matemática, la organización curricular debe tener en cuenta las habilidades necesarias del estudiantado, los conocimientos previos, el nivel de abstracción que se requiere y la naturaleza lógica de la matemática. Finalmente, los métodos de enseñanza deben estar relacionados con la institución y con el currículo de matemática, de forma tal que el lenguaje utilizado debe estar acorde a los niveles de comprensión del estudiantado, 
la secuencia de unidades didácticas a desarrollar de estar en concordancia con la lógica de la matemática, además, deben ser consideradas las diferencias individuales del estudiantado.

4. Dificultades asociadas al desarrollo cognitivo del estudiantado: el personal docente de matemática debe conocer los estadios generales del desarrollo intelectual de sus estudiantes, lo cual le permite comprender sus formas de razonamiento y las tareas matemáticas que debe plantear según sea el caso. Existen diferentes teorías generales sobre el desarrollo cognitivo, tales como el enfoque jerárquico del aprendizaje, el evolutivo, el estructuralista, el constructivista, entre otros.

5. Dificultades asociadas a las actitudes afectivas o emocionales: estas están relacionadas con los bloqueos que presentan gran cantidad de estudiantes debido al miedo y tensión provocada por la matemática. Existen factores, tales como la naturaleza jerárquica de la matemática, la actitud del profesorado, los estilos de enseñanza, las actitudes y creencias que les son transmitidas, entre otros, los cuales influyen en las actitudes negativas y de disgusto que presenta el estudiantado.

Por su parte, Radatz (1980) categoriza los errores en matemática de la siguiente manera:

1. Errores debidos a las dificultades del lenguaje: estos errores se presentan debido a la falta de comprensión semántica (significado, sentido o interpretación), por parte del estudiantado, del lenguaje matemático, lo cual desemboca en uso incorrecto de los símbolos y términos matemáticos.

2. Errores debidos a dificultades para obtener información espacial: son errores que se presentan del uso inadecuado de imágenes inadecuadas en algunas situaciones matemáticas.

3. Errores debidos al aprendizaje deficiente de habilidades y conocimientos previos: corresponden a errores causados por el deficiente manejo de contenidos y procedimientos que se suponen conocidos previamente.

4. Errores debidos a asociaciones incorrectas: son errores causados por la insuficiente capacidad del pensamiento para adaptarse a situaciones novedosas.

\section{Marco Metodológico}

\section{Tipo de investigación}

Esta investigación está dentro de la modalidad de investigación cualitativa. Su propósito es determinar la perspectiva de los principales actores en el proceso de enseñanza-aprendizaje del cálculo diferencial e integral en una variable a nivel universitario en lo concerniente a propósitos, enfoque, metodologías y dificultades en la enseñanza y aprendizaje de esta área. No se pretende realizar generalizaciones, sino poner a la luz del público lector las concepciones de esta población, de manera que se puedan generar reflexiones y acciones al respecto.

Por este motivo, las técnicas e instrumentos de recolección de datos fueron diseñados desde el enfoque cualitativo, de manera que los datos recolectados permitieran la descripción y la explicación de los objetivos de este trabajo.

\section{Participantes}

Para esta investigación se consideraron como foco de estudio tres grupos: (a) autoridades administrativas de las escuelas de matemática de la UCR, UNA, ITCR y UNED, (b) personal docente de matemática con experiencia en la enseñanza del cálculo diferencial e integral en una variable de dichas instituciones, y (c) estudiantado avanzado de la carrera de Bachillerato y Licenciatura en la Enseñanza de la Matemática de la UNA. 
La selección de las universidades participantes se realizó con base en su conocida trayectoria y prestigio en la formación de profesionales en diversas carreras, incluyendo, la formación de docentes de matemática.

En total participaron seis autoridades administrativas (dos de la UCR, dos de la UNA, uno del ITCR y uno de la UNED) con puestos tales como dirección de escuela o coordinación de departamento o área.

En cuanto al personal docente de matemática de las escuelas de matemática, se contó con 58 personas de las cuatro universidades participantes. Para la escogencia de este grupo académico, se consultó a las autoridades de cada universidad participante. Estas facilitaron una lista con los nombres del personal docente, que, según su criterio, podrían dar aportes significativos a esta investigación. El grupo de docentes participantes ha impartido al menos un curso en la línea del cálculo diferencial e integral en una variable para servicio o para la carrera de Enseñanza de la Matemática en alguna de las universidades antes mencionadas.

El grupo de estudiantado avanzado de la carrera de Enseñanza de la Matemática de la UNA, lo constituyen 25 estudiantes de cuarto o quinto año del plan de estudios de la carrera. Tienen como principal característica haber aprobado los cursos en la línea del cálculo diferencial e integral en una variable de su plan de estudios.

\section{Técnicas e instrumentos de recolección de datos}

Se utilizaron tres técnicas para la recolección de datos: (a) una entrevista a las autoridades administrativas de las escuelas de matemática de las universidades estatales participantes, (b) un cuestionario dirigido al personal docente de matemática de las escuelas de matemática, y (c) un taller con estudiantes de la carrera de Enseñanza de la Matemática de la UNA. Estas técnicas se aplicaron durante el segundo ciclo del 2015.

Para la entrevista a autoridades se elaboró una lista de preguntas semi-estructuradas que guiaron las discusiones del equipo investigador con las autoridades. Los temas tratados fueron el objetivo del cálculo diferencial e integral y la metodología empleada en los cursos de formación de futuros docentes de matemática y para otros grupos profesionales. Además, se consultó sobre la utilización de resolución de problemas como metodología de enseñanza en los cursos de cálculo diferencial e integral; y los temas que presentan mayor dificultad en la enseñanza-aprendizaje del cálculo diferencial e integral. Las entrevistas fueron grabadas con el objetivo de complementar y triangular la información obtenida.

El cuestionario al personal docente constaba de cinco apartados: (a) información general del sujeto encuestado, (b) resolución de problemas en la enseñanza-aprendizaje de la matemática, (c) propósitos y enfoques en la enseñanza-aprendizaje del cálculo diferencial e integral en una variable, (d) tipos de dificultades y errores en la enseñanza del cálculo diferencial e integral en una variable, (e) temas y contenidos de mayor dificultad en la enseñanza del cálculo diferencial e integral en una variable. Cada apartado estaba constituido de preguntas cerradas y una o dos preguntas abiertas. Los ítems incluyeron escalas de Likert y de ordenación.

Para el taller con estudiantes se elaboró una lista de preguntas generadoras sobre los propósitos del cálculo diferencial e integral en la formación de futuras generaciones de docentes de matemáticas, sobre el enfoque de enseñanza y la metodología recibidos en los cursos de cálculo y los que consideraban más apropiado. Estas preguntas guiaron la discusión de los subgrupos de trabajo y la discusión grupal. La información por subgrupo fue recopilada de forma escrita con el instrumento de preguntas generadoras que se les entregó y la plenaria fue grabada para su posterior análisis.

\section{Resultados}

Los resultados se presentan a partir de tres categorías. Estas destacan los propósitos, el enfoque y metodología de los cursos de cálculo diferencial e integral en una variable y el grado de dificultad de los contenidos en esta línea, a partir de la información proporcionada por los grupos participantes. 
Perspectivas de las autoridades, personal docente y estudiantes de la carrera sobre los propósitos de la enseñanza del cálculo diferencial e integral en una variable para preparación de docentes de matemática en formación inicial

Los sujetos participantes coinciden en que los principales propósitos del cálculo diferencial e integral en una variable en la formación de futuro personal docente de matemática son: (a) desarrollar habilidades y competencias específicas, (b) establecer bases teóricas para cursos posteriores, (c) desarrollar herramientas para la resolución de problemas, y (d) desarrollar herramientas para una mejor labor de aula.

Afirman que la línea del cálculo debe permitirles, a las futuras generaciones de docentes de matemática, abstraer propiedades estructurales de objetos matemáticos de manera que puedan comprenderlas mediante una demostración o refutarlas con contraejemplos. Manifiestan que los contenidos de los cursos en esta línea deben favorecer los procesos demostrativos, deductivos y de abstracción mediante el análisis del comportamiento de funciones reales de variable real, el estudio del infinito, entre otros aspectos. Estos deben facilitar la comprensión y uso del lenguaje matemático al estudiantado, y para que este proponga, analice e interprete modelos de situaciones reales utilizando los conocimientos adquiridos.

Aseguran que el cálculo es fundamental para la comprensión de otros temas matemáticos de las distintas áreas disciplinarias de los planes de estudios de preparación de docentes de matemática en formación inicial.

Los contenidos del cálculo diferencial e integral pueden enfocarse de manera que se generen herramientas de demostración, las cuales pueden ser útiles para cursos posteriores.

Destacan la importancia del cálculo diferencial e integral en la preparación de docentes de matemática en formación inicial para un buen desempeño en proyectos de secundaria tales como Matemática para la Enseñanza Media (MATEM) y el bachillerato internacional. Coinciden en que el cálculo debe enriquecer la visión del futuro docente sobre la matemática y su utilidad en otras ciencias.

El personal docente y estudiantes de la carrera agregan a los propósitos anteriores la importancia del cálculo como referente para el estudio del desarrollo histórico de la matemática. Consideran que los cursos de cálculo deben permitir la exploración y discusión del desarrollo histórico de algunos conceptos fundamentales del cálculo y, por ende, de la matemática. Según estos grupos, la línea del cálculo debe permitir, a las futuras generaciones docentes, conocer y comprender las demostraciones de algunos teoremas clásicos importantes en distintas áreas de la matemática.

\section{Perspectivas de las autoridades, personal docente y estudiantes de la carrera sobre el enfoque y la metodología de la enseñanza del cálculo diferencial e integral en una variable en la formación de futuras generaciones de docentes de matemática}

En cuanto al enfoque de los cursos de cálculo diferencial e integral en una variable, el estudiantado de la carrera identifica dos direcciones opuestas en su formación en esta línea. Por un lado, el enfoque práctico en donde el desarrollo del curso estaba fundamentado en la resolución de ejercicios rutinarios. En cuanto a la teoría, se enunciaban los teoremas, sin su demostración. Por otro lado, el enfoque teórico, en donde se hacía la demostración de la mayoría de los teoremas y se presentaban pocos problemas de aplicación. La variante en el enfoque ha dependido, afirman, del docente o la docente que impartiera el curso.

Aseguran que la metodología predominante en estos cursos fue la clase magistral, con poca participación por la parte estudiantil; y los pocos recursos utilizados consistían en las notas de clase de cada docente que impartía el curso o algún libro de texto. Las estrategias de evaluación predominantes fueron las pruebas escritas.

Coinciden que en los cursos de cálculo diferencial e integral debe existir un equilibrio entre la teoría y la práctica. En este sentido, consideran las demostraciones fundamentales, pero deben acompañarse 
de ejercicios de aplicación. Proponen el uso de la tecnología como herramienta didáctica, actividades de laboratorio y proyectos de investigación.

Las autoridades, por su parte, reconocen un ausentismo de diversidad en cuanto a las estrategias metodológicas en los cursos de cálculo. Coinciden con el estudiantado en que la clase magistral es la única estrategia presente; y aunque afirman que esta favorece la presentación de algunos fundamentos teóricos, consideran necesario acompañarla de otras estrategias didácticas, las cuales pueden variar en función de los temas a desarrollar.

Ambos grupos, estudiantes y autoridades, proponen la resolución de problemas como estrategia complementaría, pero no de la forma tradicional. En este sentido, consideran importante el planteamiento de problemas no rutinarios, los cuales permitan, como parte del proceso de resolución, el desarrollo de un concepto determinado. Es importante que los problemas tengan múltiples soluciones. Aseveran que la historia de la matemática es un recurso importante, pues permite al estudiantado conocer el proceso constructivo del conocimiento a través del tiempo. Consideran conveniente el desarrollo de pequeñas investigaciones sobre algunos de los conceptos en estudio, la elaboración de unidades didácticas y presentaciones en donde se lleven a cabo procesos de evaluación colaborativa. Manifiestan que se debe utilizar la modelización o simulación como metodología de enseñanza-aprendizaje; en este sentido, es importante mostrar situaciones en donde el cálculo diferencial e integral en una variable se utilice para la resolución de un problema dado. Encuentran importante el uso de herramientas tecnológicas como un apoyo a la docencia.

Por otra parte, la mayoría de docentes coincide en que los contenidos que se imparten en los cursos de cálculo diferencial e integral en una variable, en una carrera de Enseñanza de la Matemática, deben desarrollarse en una secuencia lógica y de manera rigurosa a nivel matemático. Una minoría considera que deben desarrollarse de manera articulada en función de la resolución de un problema histórico o de la vida cotidiana, o en secuencia determinada por su génesis histórica.

El grupo de docentes considera que la metodología para la enseñanza en los cursos de cálculo diferencial e integral en una variable en una carrera de Enseñanza de la Matemática debe incluir: sesiones de resolución de problemas, sesiones de discusión y reflexión, presentaciones magistrales por parte del personal docente, sesiones de laboratorio y presentaciones magistrales por parte de estudiantes.

Respecto a los recursos didácticos que deben utilizarse, el personal docente propone: la pizarra, libros de texto, el uso de algún software computacional, los manipulativos y la calculadora graficadora.

Finalmente, el personal docente plantea como métodos de evaluación las pruebas escritas, las presentaciones de temas o ejercicios, los proyectos, los portafolios y los diarios reflexivos.

\section{Perspectivas de las autoridades, personal docente y estudiantes de la carrera sobre los temas de mayor dificultad en el aprendizaje del cálculo diferencial e integral en una variable en la formación de futuro personal docente de matemática}

En relación con las dificultades en el proceso de enseñanza-aprendizaje del cálculo diferencial e integral en cursos de Enseñanza de la Matemática, las autoridades las asocian principalmente a:

1. Dificultades debidas al desarrollo teórico de los contenidos: afirman que los cursos de cálculo diferencial e integral para las carreras de enseñanza de la matemática tienen un enfoque predominantemente teórico, donde se hace una presentación formal de los conceptos y se realizan las demostraciones de la mayoría de los resultados expuestos. Esto eleva el nivel de abstracción de los cursos lo que genera dificultades a sus estudiantes.

2. Dificultades asociadas a la comprensión e interpretación: estas dificultades se presentan en la resolución de problemas de optimización y de razones de cambio relacionadas. Al estudiantado se le dificulta comprender lo que se le plantea en un problema dado, de manera que no logra 
determinar los elementos esenciales. El estudiantado no está habituado a plantear y resolver problemas, aunque tenga la teoría necesaria para hacerlo.

3. Dificultades asociadas a la identificación de patrones: al estudiantado se le dificulta identificar la técnica de integración apropiada para calcular una integral indefinida. Sus deficiencias para observar y encontrar patrones dificultan este aprendizaje y termina haciéndolo de forma mecánica.

El personal docente, por su parte, atribuye tales dificultades a la poca comprensión de parte del estudiantado del lenguaje matemático, la deficiencia en el manejo de los conocimientos previos, la falta de habilidades matemáticas tales como observar patrones, conjeturar, generalizar e inferir; y la falta de abstracción y visualización del estudiantado.

Otras dificultades que una parte del personal docente mencionó fueron:

1. Los aspectos socioeconómicos: los problemas en esta área pueden afectar las condiciones en las que estudiantes atienden la clase.

2. La disciplina de estudio de estudiantes: estudiantes que carecen de un horario de estudio adecuado, no asisten a las horas de consulta, no utilizan los materiales didácticos sugeridos.

3. Los aspectos afectivos: la falta de motivación de parte de estudiantes para el estudio, la poca empatía docente con estudiantes y viceversa.

4. La orientación vocacional: estudiantes que ingresan a una carrera sin tener las habilidades adecuadas para afrontarla.

5. Los aspectos didácticos: la falta de tiempo para madurar los contenidos, la poca actualización de docentes.

Identifican como los contenidos con mayor dificultad en el proceso de enseñanza y aprendizaje en los cursos de cálculo diferencial e integral para la formación de docentes de matemática:

1. Límites de funciones reales de variable real: Demostración de los teoremas fundamentales sobre límites infinitos, al infinito e infinito al infinito, definición formal de límite de una función real de variable real y demostración de teoremas básicos sobre límites.

2. Continuidad de funciones reales de variable real: Continuidad uniforme, demostración de los teoremas del máximo-mínimo, de los valores intermedios, de la preservación de intervalos y de los teoremas básicos sobre continuidad, la función inversa continua.

3. Derivación de funciones reales de variable real: Demostración de los teoremas básicos sobre las derivadas tales como las derivadas de sumas, productos, cocientes y la regla de la cadena, además de las demostraciones de los teoremas de Darboux o del valor intermedio para derivadas, el teorema de Rolle y el del valor medio. Los teoremas de Rolle, valor medio y sus consecuencias tales como el crecimiento de una función mediante el estudio de su derivada.

4. Integración definida de funciones reales de variable real: Demostración del primer y segundo teoremas fundamentales del cálculo y del teorema del valor medio para integrales. Las integrales inferiores y superiores de Riemann, las funciones Riemann integrables en un intervalo cerrado, la condición de Riemann y las sumas superiores e inferiores de Darboux para funciones acotadas.

5. Integración definida de funciones reales de variable real: Los métodos de integración mediante sustitución trigonométrica y de integración de fracciones racionales de senos y cosenos mediante la sustitución tangente del ángulo medio.

6. Sucesiones numéricas: Demostraciones de los teoremas básicos de sucesiones, la demostración del teorema de Bolzano-Weierstrass, las sucesiones de Cauchy y las subsucesiones y el teorema de Bolzano-Weierstrass. 
7. Series numéricas: Criterios de convergencia para series numéricas: criterio de la condición necesaria, criterio de comparación directa, criterio de comparación mediante el límite, criterio del cociente, criterio de la raíz, criterio de la integral y las p-series, criterio de las series alternantes. Convergencia absoluta y condicional.

8. Series de potencias: Demostración del teorema de la existencia del radio de convergencia, la representación de funciones por series de potencia, la fórmula de Taylor con resto de Lagrange y las aplicaciones a cálculos aproximados de la fórmula de Taylor.

9. Aplicaciones de la derivada: Los problemas de ritmos relacionados y de optimización.

10. Aplicaciones de la integral: Cálculo de volúmenes de sólidos de revolución: método de discos y método de capas.

11. Integrales impropias: Criterios de convergencia para integrales impropias: criterio de la condición necesaria, criterio de Cauchy, criterio de Weiertrass, criterio de comparación directa, criterio de comparación mediante límite. Convergencia absoluta y condicional.

Por otro lado, el grupo de estudiantes menciona que los contenidos que mayores dificultades le presentaron en su proceso de aprendizaje fueron: los problemas de optimización y de razones de cambio relacionadas, series y sucesiones, la continuidad de funciones reales de variable real y los sólidos de revolución. Atribuyen dichas dificultades al enfoque del curso y a las estrategias metodológicas utilizadas por el personal docente.

\section{Conclusiones}

El personal docente de matemática no es matemático puro, ni ingeniero, ni pedagogo general. Por tal razón, su formación matemática debe poseer elementos distintos a la formación de otras profesiones que tienen dentro de su plan de estudios cursos de matemática. Específicamente, los principales actores del proceso de enseñanza-aprendizaje de esta línea concuerdan en que el cálculo diferencial e integral debe aportar, en la formación de docentes de matemática, conocimientos, habilidades y competencias acordes con su quehacer, tales como el desarrollo del pensamiento lógico-matemático, la abstracción, la comprensión, el análisis de modelos matemáticos, el uso del lenguaje matemático, la deducción y la demostración, entre otros. Particularmente, los elementos históricos del cálculo deben ampliar su visión sobre los propósitos de la matemática en la resolución de problemas de la vida real, así como brindarle elementos que favorezcan la labor de aula.

Los cursos de cálculo diferencial e integral en una variable para preparación de docentes de matemática en formación inicial que se ofrecen en la Universidad Nacional (UNA), la Universidad de Costa Rica (UCR), el Instituto Tecnológico de Costa Rica (ITCR) y la Universidad Estatal a Distancia (UNED), son similares en cuanto a los contenidos a desarrollar, el enfoque, las metodologías, los recursos didácticos y los métodos de evaluación. Los resultados revelan una tendencia por el enfoque de los cursos teórico-formales, con pocos elementos prácticos y de aplicación. En relación con la metodología empleada, predomina la clase magistral con evaluaciones basadas en pruebas escritas. La resolución de problemas es considerada dentro del proceso de enseñanza-aprendizaje en la solución de ejercicios posterior al desarrollo de la clase.

Los resultados de esta investigación concuerdan con la teoría sobre las principales dificultades en el proceso de enseñanza-aprendizaje del cálculo diferencial e integral en una variable en la formación de docentes de matemática, quienes radican en la falta de habilidades matemáticas tales como la observación de patrones, la formulación de conjeturas, la generalización, la abstracción y la visualización; así como la falta de comprensión e interpretación del estudiantado. Adicionalmente, se agregan las dificultades socioeconómicas, afectivas, y las pocas disciplinas de estudio.

De lo anterior, se recomienda replantear los cursos en la línea del cálculo diferencial e integral en una variable para la formación de futuro personal docente de matemática, de manera que el enfoque 
y la metodología de los temas a desarrollar respondan al perfil de formación de tales docentes. Dicho replanteamiento debe disminuir las principales dificultades en el proceso de enseñanza y aprendizaje del cálculo, señaladas por las investigaciones en educación matemática y confirmadas por los grupos participantes de esta investigación.

Las propuestas podrían abordar, inicialmente, los temas que se han señalado como los de mayor dificultad tales como: problemas de optimización y de razones de cambio relacionadas, series y sucesiones, continuidad de funciones reales de variable real y sólidos de revolución.

\section{ReFERENCIAS}

Abrate, R., Pochulu, M. y Vargas, J. (2006). Errores y dificultades en matemática: Análisis de causas y sugerencias de trabajo. Universidad Nacional de Villa María, Buenos Aires, Argentina. Recuperado de http:// unvm.galeon.com/Libro1.pdf

Artigue, M. (1998). L' évolution des problématiques en didactique de analyse. Recherches en Didactique des Mathématiques, 18(2), 231-262.

Artigue, M. (1991). Analysis. En D. Tall (Ed.), Advanced mathematical thinking (pp. 167-198). Netherlands: Kluwer, A.

Ball, D. L., Thames, M. H., y Phelps, G. (2008). Content Knowledge for teaching: What makes it special? Journal of Teacher Education, 59(5), 389-407.

Cuevas, C. y Pluvinage, F. (2009). Cálculo y tecnología. Revista el Cálculo y su Enseñanza, 1, 45-59. Recuperado de http://mattec.matedu.cinvestav.mx/el_calculo/index.php?vol=1\&index_web=7\&index_mgzne

Delgado, M. (2009). Matemática visual: Simulaciones relativas al teorema fundamental del cálculo. Revista el Cálculo y su Enseñanza, 1, 61-74. Recuperado de http://mattec.matedu.cinvestav.mx/el_calculo/index.php? vol=18index_web=7\&index_mgzne

Díaz, M. (2009). Conocimientos de los profesores preuniversitarios de cálculo acerca del significado y las interpretaciones de la derivada. Revista el Cálculo y su Enseñanza, 1, 75-90. Recuperado de http:// mattec.matedu.cinvestav.mx/el_calculo/index.php?vol=1\&index_web=7\&index_mgzne

D’Ambrósio, U. (2002). A matemática nasescolas. Educação Matemática em Revista, 9(11), 29-33.

Depool, R. (2005). La enseñanza y aprendizaje del cálculo integral en un entorno computacional. Actitudes de los estudiantes hacia el uso de un programa de cálculo simbólico (PCS). Revista de Didáctica de las Matemáticas, 62, 3-31.

Di Blasi Regner, M. (2003). Dificultades y errores: Un estudio de caso. Comunicación breve presentada en el II Congreso Internacional de Matemática Aplicada a la Ingeniería y Enseñanza de la Matemática en Ingeniería. Buenos Aires.

Dos-Santos, E. (2012). Idoneidad de procesos de estudio del cálculo integral en la formación de profesores de matemáticas: Una aproximación desde la investigación en didáctica del cálculo y el conocimiento profesional (Tesis doctoral). Universidad de Granada, España.

Dreyfus, T. y Eisenberg, T. (1990). On difficulties with diagrams: theoretical issues. Proceedings of the four Tenth International Conference for the Psychology of Mathematics Education, 2, 27-33.

Fischbein, E. (1994). The interaction between the formal, the algorithmic and the intuitive components in a mathematical activity. Didactics of mathematics as a scientific discipline, 231-245.

Godino, J. (2010). Perspectiva de la didáctica de las matemáticas como disciplina tecnocientífica. Universidad de Granada, España. Recuperado de http://www.ugr.es/ jgodino/fundamentos_teoricos/perspectiva_ddm.pdf

Harel, G. y Trgalová, J. (1996). Higher mathematics education. A. Bishop, M.A.K. Clements, C. Keitel-Kreidt, J. Kilpatrick, C. Laborde (Eds.), International handbook of mathematics education (Part Two, pp. 675-700). Netherlands: Kluwers Academic Press. 
Ímaz, C y Moreno, L. (2009). Sobre el desarrollo del cálculo y su enseñanza. Revista el Cálculo y su Enseñanza, 1, 99-112. Recuperado de http://mattec.matedu.cinvestav.mx/el_calculo/index.php? vol=1\&index_web=7\&index_mgzne

Ministerio de Educación Pública. (2012). Programas de estudio de matemáticas I, II y III ciclos de la educación general básica y ciclo diversificado. San José, Costa Rica: Autor.

Moreno, M. (2005). El papel de la didáctica en la enseñanza del cálculo: Evolución, estado actual y retos futuros. En A. Maz, B. Gómez y M. Torralbo (Eds.), Noveno Simposio de la Sociedad Española de Educación Matemática SEIEM (pp.81-96). Universidad de Córdoba. Recuperado de http://redined.mecd.gob.es/xmlui/bitstream/ handle/11162/48280/01120112000110.pdf?sequence $=1$

Nava, M. y Reyes, A. (2009). Creencias y conocimientos acerca de precálculo y cálculo de un grupo de profesores de bachillerato. El cálculo y su enseñanza. Cinvestav del Instituto Politécnico Nacional, México D.F.

Radatz, H. (1980). Students' errors in the mathematical learning process: a survey. For the Learning of Mathematics, $1(1), 16-20$.

Ruthven, K. (2002). Linking researching with teaching: Towards synergy of scholarly and craft knowlege. En L. D. English, M. Bartolini-Busi, G. A. Jones, R. Lesh, R. and D. Tiroshm, Handbook of International research in mathematics education (pp. 581-598). London: Lawrence Erlbaum Ass.

Salinas, P. y Alanís, J. (2009). Hacia un nuevo paradigma en la enseñanza del cálculo dentro de una institución educativa. Revista Latinoamericana de Investigación en Matemática educativa, 12(3), 355-382.

Selden, J., Mason, A. y Selden, A. (1994). Even good calculus students can't solve non-routine problems. En J. Kaput y E. Dubinsky (Eds.), Research issues in undergraduate mathematics learning (pp. 19-26). United States: MAA 3.

Tall, D. (1997). Functions and Calculus. En A. Bishop, M. A. K. Clements, C. Keitel-Kreidt, J. Kilpatrick, C. Laborde (Eds.), International Handbook of Mathematics Education (pp. 289-325). Dordrecht: Kluwer.

\section{Notas}

[3] Escuela de Matemática es lo equivalente a Departamento de Matemática.

\section{BY-NC-ND}

